\title{
Tomographic flow cytometry by digital holography
}

\author{
Francesco Merola ${ }^{1}$, Pasquale Memmolo ${ }^{1}$, Lisa Miccio ${ }^{1}$, Roberto Savoia ${ }^{1}$, Martina Mugnano ${ }^{1}$, Angelo Fontana ${ }^{2}$, \\ Giuliana D'Ippolito ${ }^{2}$, Angela Sardo ${ }^{2}$, Achille Iolascon ${ }^{3}$, Antonella Gambale ${ }^{3}$ and Pietro Ferraro ${ }^{1}$
}

\begin{abstract}
High-throughput single-cell analysis is a challenging task. Label-free tomographic phase microscopy is an excellent candidate to perform this task. However, in-line tomography is very difficult to implement in practice because it requires a complex set-up for rotating the sample and examining the cell along several directions. We demonstrate that by exploiting the random rolling of cells while they are flowing along a microfluidic channel, it is possible to obtain in-line phase-contrast tomography, if smart strategies for wavefront analysis are adopted. In fact, surprisingly, a priori knowledge of the three-dimensional position and orientation of rotating cells is no longer needed because this information can be completely retrieved through digital holography wavefront numerical analysis. This approach makes continuous-flow cytotomography suitable for practical operation in real-world, single-cell analysis and with a substantial simplification of the optical system; that is, no mechanical scanning or multi-direction probing is required. A demonstration is given for two completely different classes of biosamples: red blood cells and diatom algae. An accurate characterization of both types of cells is reported, despite their very different nature and material content, thus showing that the proposed method can be extended by adopting two alternate strategies of wavefront analysis to many classes of cells.
\end{abstract}

Light: Science \& Applications (2017) 6, e16241; doi:10.1038/lsa.2016.241; published online 7 April 2017

Keywords: microfluidics, red blood cells, tomographic microscopy, wavefront analysis

\section{INTRODUCTION}

To date, one of the most powerful imaging tools for analyzing biological samples is tomography, which is able to furnish complete characterizations in three-dimensional $(3 \mathrm{D})^{1}$. Various classes of tomographic concepts exist, such as X-ray computed tomography, optical coherence tomography $\mathrm{y}^{2,3}$ and tomographic phase microscopy $(\mathrm{TPM})^{4-14}$. TPM is based on quantitative phase imaging techniques for the accurate 3D refractive index (RI) mapping of cells, as demonstrated by Feld's group at $\mathrm{MIT}^{4}$, or even by white light sources applied to red blood cells (RBCs), as shown by Popescu's group ${ }^{7,8}$. Park and coworkers demonstrated a reconstruction of a 3D RI distribution employing sparse angle illumination ${ }^{5,9,11}$. Ozcan's group proposed a lens-free holographic microscope, which enables the imaging of a very large volume (tens of $\mathrm{mm}^{2}$ ) and the possibility to perform an optical in-flow tomography by exploiting a pixel superresolution technique ${ }^{12-14}$. Recently, Psaltis' group proved a smart learning method for building tomographic structures ${ }^{15,16}$. TPM setups require the sample to be observed along different directions with respect to the probing beam. Recording is often accomplished by adopting beam deflection ${ }^{5,14,17}$; however, in this case, some problems arise because the angles are limited to $150^{\circ}$, thus affecting the accuracy of the tomographic reconstruction. Alternatively, direct mechanical rotation of the sample ${ }^{6}$ is problematic because mechanically manipulating the biological specimen introduces the risk of altering the sample. Tomographic arrangements occasionally use holographic optical tweezers $^{18}$ to rotate the sample without mechanical contact $^{19-21}$; unfortunately, they do not allow high-throughput and in-line useful conditions for continuous in-flow tomography. In another case, Heng et al. ${ }^{22}$ adopted an optofluidic microscope, for which the flow was used to provide a scanning mechanism; however, the sample rotation was considered an undesired effect.

In summary, to date, all tomographic methods require a highprecision, opto-mechanical and/or optoelectronic device to acquire a set of many images by probing the sample along a large number of controlled directions. Moreover, to successfully apply the tomography algorithms, each recorded image must be tagged with the correct cell's rotation angle with respect to the probing beam, thus requiring robust and effective control of the angular positions. Overcoming such difficulties represents the main critical issue in all tomographic approaches and, in practice, limits the implementation of tomography, especially at the microscopic scale, because the current approaches are very cumbersome and unsuitable for widespread use in the life sciences. Here we present a technological improvement by demonstrating an in-line TPM approach that simplifies the optical set-up, thus opening a route for a real-world tool in the life sciences for single-cell analysis in continuous flow. Essentially, we show for the first time that the wavefront transmitted by the cells intrinsically carries information beyond the optical path lengths (OPLs) to obtain

${ }^{1}$ CNR-ISASI, Istituto di Scienze Applicate e Sistemi Intelligenti 'E. Caianiello', CNR—Consiglio Nazionale delle Ricerche, Pozzuoli 80078, Italy; ${ }^{2}$ CNR-ICB, Istituto di Chimica Biomolecolare, Pozzuoli 80078, Italy and ${ }^{3}$ Department of Molecular Medicine and Medical Biotechnology, University of Naples Federico II \& CEINGE-Advanced Biotechnologies, Napoli 80145, Italy

Correspondence: P Ferraro, Email: pietro.ferraro@cnr.it

Received 4 May 2016; revised 3 October 2016; accepted 10 October 2016; accepted article preview online 17 October 2016 
quantitative phase-contrast maps (QPMs). In fact, we prove that a wavefront transmitted through the cell also contains information on the value of its angular position with respect to the direction of the probing laser beam. We explain below how the angles can be extracted numerically from the QPMs by intelligent numerical processing through two different strategies, thus allowing TPM that is suitable for all types of cells by using a single laser beam without mechanical or electro-optical scanning.

The two strategies for angle recovery are conceived on the basis of the inner structure of cells. In particular, the rolling angles of cells with a homogeneous RI distribution are calculated by exploiting the biolens effect $^{23}$. Alternatively, rolling angles of cells with inhomogeneous RI are obtained by observing the QPMs' mirror symmetry around the axis of rotation.

The proposed innovative approach has been tested with two different classes of biological samples having relevant impact in our life, that is, $\mathrm{RBCs}^{24,25}$ (with homogeneous RI) and diatoms algae $(\mathrm{DAs})^{26,27}$ (with inhomogeneous RI). Note that RBCs and DAs have completely different external shapes and inner structures, although they are all quite simple. Moreover, RBC and DA are made of diverse materials.

Although RBCs and DAs are very different from a biological perspective, they have some features in common. In fact, RBCs and DAs have a crucial role in the health of the human body and our planet, respectively. Moreover, these materials are characterized by their high abundance: RBCs account for nearly a quarter of the total number of cells in the human body, and diatoms are responsible for over $40 \%$ of the photosynthesis that occurs in the world's oceans, and without them, the ocean would be unable to support the amount of life that it does. Furthermore, diatoms are useful tools for monitoring environmental conditions and are commonly used in studies of water quality, and the morphology and content of RBCs (that is, hemoglobin) are important biomarkers for many severe blood diseases ${ }^{28}$.

An accurate study of the full 3D structure and the content of such diverse types of cells is of vital importance for human health (regarding RBCs) and for the earth's ecosystem (regarding diatoms); as a result, a simple diagnostic tool that can operate in a highthroughput mode is highly desirable to, for example, find and identify rare cells in blood or contaminants in oceans.

Here we exploit the random self-rotation of the cells while they are flowing along microfluidic channels ${ }^{29-31}$, thereby avoiding the direct rotation of the cells or the use of laser-beam angular scanning. Furthermore, we demonstrate that a priori knowledge of the rotation angle in $3 \mathrm{D}$ is no longer necessary because it can be accurately retrieved from the intelligent processing of the QPMs. The only requirement is to have one full angular revolution of the cells inside the field of view of the microscope while they are flowing. We named this proposed approach Rolling-TPM (R-TPM). Moreover, this approach allows fast single-cell analysis in high-throughput modality because no mechanical or electro-optical angular scanning of the laser beam is required.

Regarding $\mathrm{RBCs}^{32,33}$, we demonstrate their full characterization in terms of several metrics, such as 3D morphology, corpuscular hemoglobin $(\mathrm{CH})$, volume $(V)$ and RI. Moreover, we demonstrate both identification and sorting capabilities for anemia blood disease, in which some of the RBCs parameters differ slightly from healthy ones. Regarding DA, we instead show the capability to obtain the outer structure and the detailed 3D inner structure of auto-fluorescent chloroplasts via whole R-TPM imaging without the recurring use of fluorescent imaging.

\section{MATERIALS AND METHODS}

\section{Blood preparation and isolation of erythrocytes}

Human blood $(\sim 4.0 \mathrm{~mL})$ was collected in a 7.2-mg $\mathrm{K}_{2}$ EDTA vacutainer tube (BD, Plymouth, UK) from a healthy volunteer. Blood was centrifuged at room temperature at 2500 r.p.m. for $15 \mathrm{~min}$ to separate RBCs at the bottom of the sterile centrifugation tube from the plasma and buffy coat. After centrifugation, the plasma and buffy coat were discarded, and the RBC pellet $(\sim 1.5 \mathrm{~mL})$ was washed with a saline solution of $0.90 \% \mathrm{w} / \mathrm{v}$ of sodium chloride $(\mathrm{NaCl})$ in sterile water in a 1:1 ratio and re-centrifuged at room temperature at 2500 r.p.m. for $10 \mathrm{~min}$. After the second centrifugation, the supernatant fraction was removed, and an aliquot of isolated RBCs $(\sim 100 \mu \mathrm{L})$ was diluted in $10 \mathrm{~mL}$ of the saline solution of $0.90 \% \mathrm{w} / \mathrm{v}$ of $\mathrm{NaCl}$ in sterile water with a final osmolarity of $308 \mathrm{mOs} \mathrm{mL}^{-1}$ to maintain the osmotic pressure of the RBCs. For the experiments, a final volume of diluted RBCs $(\sim 100 \mu \mathrm{L})$ was used. Altered RBC shapes were obtained by changing the buffer osmolarity, and a buffer of 205 and $410 \mathrm{mOs} \mathrm{mL}^{-1}$ was used to perform experiments under hypotonic and hypertonic conditions, respectively.

\section{Diatoms preparation}

The two strains of diatoms (T. rotula CCMP 3264 and S. marinoi CCMP 2092) were purchased from the National Center for Marine Algae and Microbiota (Bigelow Laboratory for Ocean Sciences, East Boothbay, ME, USA). Microalgae were cultured in $75 \mathrm{~cm}^{2}$ flasks in sterile $\mathrm{f} / 2$ medium at $20.0 \pm 1.0^{\circ} \mathrm{C}$. The artificial light illumination ( $100 \mu \mathrm{mol}$ photons $\mathrm{m}^{-2} \mathrm{~s}^{-1}$ ) was provided by daylight fluorescent tubes with a 14:10 h light:dark photoperiod. Then, $5-\mathrm{mL}$ subsamples of each culture were collected with serological pipettes under the fume hood and transferred to $15-\mathrm{mL}$ falcons. Highly concentrated cultures were diluted with filtered $(0.22 \mu \mathrm{m})$ sterile seawater and maintained at room temperature until the analyses/measurements were performed.

\section{Holographic recording and reconstructions}

We built an optofluidic platform (depicted in Figure 1) to provide high-resolution images of both flowing and rotating samples. The DH modulus is made with a $400 \mathrm{~mW}$ fiber-coupled laser at $532 \mathrm{~nm}$ acting as the source for a Mach-Zehnder interferometer, whose main beam (in green) is directed into a customized inverted microscope equipped with a water-immersion $60 \times$ objective, numerical aperture 1.20 microscope objective, thus allowing bright-field imaging of the cells. The reference beam (also in green) is recombined with the first beam by a beam splitter generating interference fringe patterns (the digital holograms) of the samples that are recorded by a $2048 \times 2048$ CCD camera (USB $3.0 \mathrm{u}$-eye, from IDS), recording at $75 \mathrm{fps}$ in full frame. The optical lateral resolution is $\sim 360 \mathrm{~nm}$, and the axial resolution is $\sim 170 \mathrm{~nm}^{34}$. In our case, different from Cotte et al. ${ }^{34}$, we are limited by the minimum detectable angle of rotation, that is, $\sim 1^{\circ}$ for this optical set-up.

The holographic recordings and reconstructions ${ }^{33}$ occur as follows. First, through a digital holography apparatus, several out-of-focus digital holograms of cells (obtained while the cells are tumbling in the microfluidic channel) are acquired for different angular positions of the cell. Next, for each acquired hologram, the corresponding QPI is numerically calculated via the angular spectrum technique ${ }^{35}$, which is a standard propagation algorithm that reconstructs the complex wavefield in terms of both amplitude and phase diffracted by the object at a certain distance from the hologram plane. The channel (by Microfluidic Chip Shop) is made of PMMA polymer and has dimensions of $1000 \mu \mathrm{m} \times 200 \mu \mathrm{m}$ (width $\times$ height). At the bottom, the cover lid is $140 \mu \mathrm{m}$ thick, which allows the use of the oil-immersion 
a

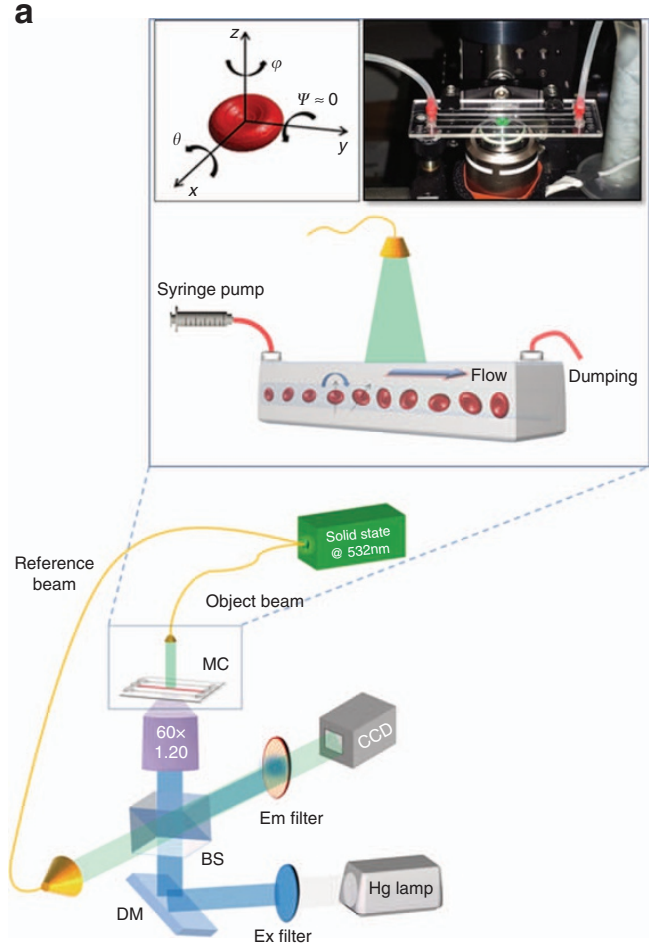

b

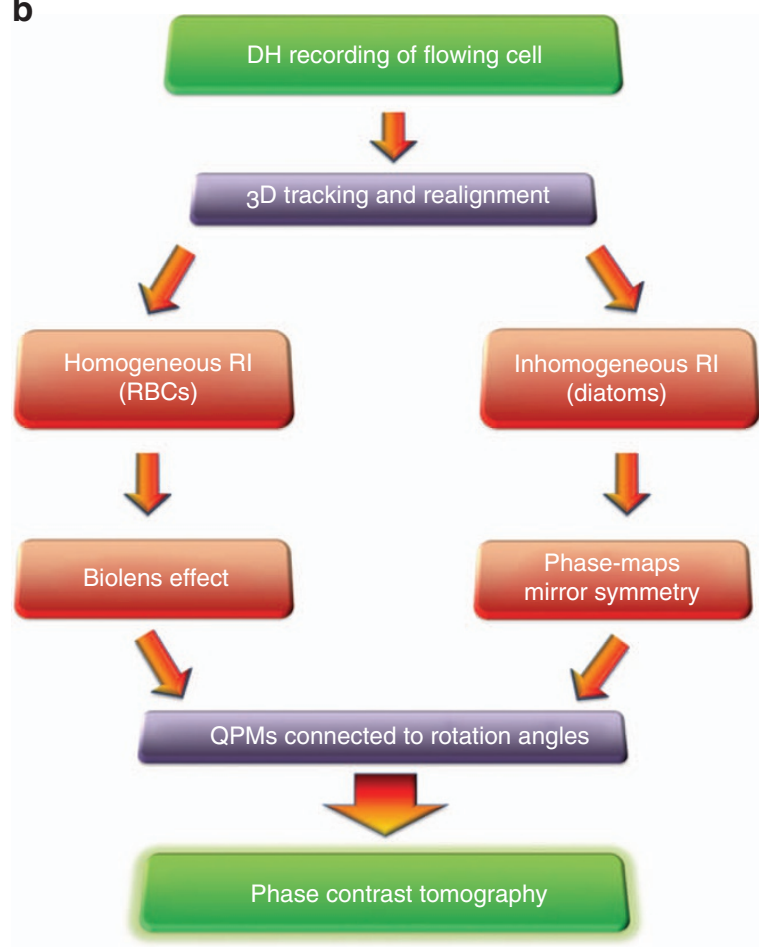

Figure 1 Working principles of the R-TPM approach. (a) Sketch of the experimental R-TPM set-up. Cells are injected into a microfluidic channel and tumble while flowing along the $y$-axis (inset of a). At the same time, a holographic image sequence is acquired. In the top-left corner of the inset, the reference system for cell tumbling is reported; in the top-right corner, a photo of the real set-up is shown. Rotation occurs around the $x$ and $z$ axes. BS, beam splitter; DM, dichroic mirror; MC, microchannel. (b) Flow chart representing the main steps of the two proposed algorithms for angle recovery and tomographic reconstruction.

objective. The sample is injected into the channel by a syringe and capillary tubes furnished by the Microfluidic Chip Shop. For the experiments with diatoms, we added a fluorescence modulus (Figure 1a). Light from a fluorescence lamp (X-cite series $120 \mathrm{pc}$ Lumen Dynamics) is directed onto the sample (blue path); a combination of excitation (GFP) and emission (Tritc) filters, which are suitable for detecting the diatom's chlorophyll, is used, together with a dichroic mirror.

\section{Fluid dynamic conditions for cells tumbling}

After the preparation is completed, the cells are injected into a microfluidic channel by a syringe pump. In our experiment, the deformation of the RBCs is negligible. Theoretical studies and numerical simulations by G Gompper and coworkers describe the different ways that an RBC moves inside a microchannel, depending on the confinement and flow strength ${ }^{36}$. In our experiment, with a very small confinement and flow strength, we observe the tumbling condition; that is, each RBC undergoes a rotation and can be approximated by a rigid body. By estimating the number of cells accomplishing a complete rotation $\left(360^{\circ}\right)$ in the field of view, we observe more than 150 rolling cells per minute. This throughput is several times larger than that obtained with classical TPM methods 9 .

\section{Tomographic reconstruction}

We use optical projection tomography ${ }^{37}$, where the inputs of the filtered back-propagation algorithm are the aligned-oriented QPMs, and the rotation angles are around the $x$-axis, $\theta$. The number of QPMs for each imaged cell in the field of view is $\sim 200$ for our camera with a frame rate of $75 \mathrm{fps}$. If the number of recordings decreases, then the resolution becomes worse, but the final shape reconstructions can be overlapped up to a lower recording limit of $\sim 80$ holograms. The calculation of the slices, which corresponds to the RI distribution of the sample along the planes orthogonal to the plane $x-y$, is performed through the inverse Radon transform. More specifically, for a given coordinate of the rotating axis in the plane $x-y$, the corresponding values of the QPMs along the orthogonal direction to the $x$-axis are collected for all rotation angles and are used together to calculate the corresponding slices by using the inverse Radon transform. Finally, these slices are joined together and processed to obtain a tomographic representation of the cell, that is, the 3D RI distribution. All numerical processing is performed off-line. By considering $\sim 200$ images, the angle recovery step requires a computational time of $11.2 \mathrm{~s}$ for RBCs and $16.8 \mathrm{~s}$ for DAs, and the projection tomography step requires 1.8 and $4.2 \mathrm{~s}$ for RBCs and DAs, respectively. Video-rate processing in tomographic phase microscopy has been recently demonstrated by using Nvidia's CUDA C platform ${ }^{38}$. An informal high-level description (that is, pseudo-code) of the operating principle of the whole tomographic reconstruction process, including the holographic $3 \mathrm{D}$ tracking step, rotating angle calculation using the two proposed methods and tomographic reconstruction algorithm, is reported in the Supplementary Information.

\section{RESULTS AND DISCUSSION}

The working conditions and the adopted optical system are depicted in Figure 1a. Cells tumble while flowing along a microfluidic chip probed by a single fixed laser beam. A second beam is used as a reference to generate interference fringes on the CCD camera. More details regarding the optical set-up are given in Materials and methods. 
Hundreds of cells per minute have been analyzed; for each one, the hologram's sequence is recorded, and the corresponding QPMs is retrieved. First, 3D holographic tracking is performed to re-align each cell with respect to its center of mass ${ }^{39-41}$. Next, the rotation angles are numerically estimated using two strategies, the choice of which depends on the type of the cell under analysis. In Figure 1b, we report the conceptual block diagram summarizing the main steps of the two proposed strategies. In particular, the cells are classified according to their RI distribution (homogeneous and inhomogeneous). By this taxonomy, almost all classes of cells can be tagged, and the performance of the two proposed algorithms depends on their biophysical features. To demonstrate the effectiveness of the two proposed strategies, we focus on RBCs as cells with homogeneous RI and DAs as cells with a more complex inner structure, that is, with inhomogeneous RI.

Specifically, RBCs can be modeled as optofluidic microlenses ${ }^{23}$; thus, Zernike polynomials are used to quantify their aberrations. The flow direction occurs along the $y$-axis (see the reference coordinates system in Figure 1a), thus allowing the contribution of rotation angle $\psi$ to be neglected. The other two orientations $(\theta$ and $\varphi)$ are calculated by aberration analyses using Zernike fitting and are computed for each QPM. We reveal here that the trend of the focus shift term $C_{4}$ can be fitted by the square cosine function of the rotation angle $\theta$ and that the orientation $\varphi$ can be retrieved from the tilt terms $C_{1}$ and $C_{2}$.

$$
\operatorname{QPM}(\mathbf{r})^{i(\theta)}=\sum_{k} C_{k}^{i(\theta)} Z_{k}(\mathbf{r}) \rightarrow C_{4} \propto \cos ^{2}(\theta) ; \phi \approx \tan ^{-1}\left(C_{1} / C_{2}\right)
$$

In Equation (1), $\mathbf{r}$ is the spatial coordinates vector, $i(\theta)$ denotes the $i$ th QPM and corresponds to a specific rotating angle $\theta, Z_{k}$ is the Zernike basis functions and $C_{k}$ is the corresponding Zernike coefficients. Each QPM is re-oriented to the first one of an angle equal to $-\varphi$.

For cells that have complex inner structures, such as DAs, we exploit an alternate intelligent image processing strategy. In fact, such cells have a detectable RI inner structure. It can be argued that a spatial symmetry exists in the reconstructed QPMs as a function of rotation angle. In particular, each QPM is compared with all others in the reconstruction sequence by searching for a twin-mirror QPM that maximizes their spatial correlation coefficient (SCC) because in these two directions, the OPLs should be equal for each pair of mirror pixels. Specifically, when the SCC of two QPMs is $>0.95$, if $\theta$ is in the range $\left[0^{\circ}, 90^{\circ}\right]$, then we assign $\theta$ and $180^{\circ}-\theta$ as the rotating angles, whereas if $\theta$ is in the range $\left[180^{\circ}, 270^{\circ}\right]$, then we assign $\theta$ and $540^{\circ}-\theta$ as the rotating angles.

$$
\left\{\begin{array}{l}
\operatorname{QPM}(\mathbf{r})^{i(\theta)} \approx \operatorname{QPM}(-\mathbf{r})^{j\left(180^{\circ}-\theta\right)} \text { if } \theta \in\left[0^{\circ}, 90^{\circ}\right] \\
\operatorname{QPM}(\mathbf{r})^{i(\theta)} \approx \operatorname{QPM}(-\mathbf{r})^{j\left(540^{\circ}-\theta\right)} \text { if } \theta \in\left[180^{\circ}, 270^{\circ}\right]
\end{array} \rightarrow \operatorname{SCC}\left(\operatorname{QPM}(\mathbf{r})^{i}, \mathrm{QPM}(-\mathbf{r})^{j}\right) \approx 1\right.
$$

In Equation (2), $i$ and $j$ with $j>i$ are the $i$ th and $j$ th QPMs, respectively. Because not all QPMs may have a corresponding mirror QPM, we tag the remaining QPMs by assuming uniform angular rotation.

Finally, we use the optical projection tomography method to calculate the 3D RI distribution of the sample ${ }^{32}$, where the inputs of the filtered back-propagation algorithm are the aligned-oriented QPMs and the rotation angles around the $x$-axis, $\theta$. Complete proofs of both mathematical relationships for retrieving the angles are reported in the Supplementary Information together with the holographic 3D tracking algorithm.

The results of R-TPM, as applied to RBCs, are reported in Figure 2 and Supplementary Movie 1. Four interesting cases are revealed. In particular, we examined both healthy (Figure 2a and 2b) and pathological (Figure 2c and 2d) RBCs, such as iron deficiency anemia and thalassemia, which are two highly diffused blood disorders. Specifically, some of the QPMs with the corresponding measured angles are illustrated on the top row of each panel of Figure 2. The inner plots show the rotating angle recovery approach, which was obtained from Equation (1). The final cytotomography results are displayed on the bottom side together with a picture of the correspondent central slices ( $z=0$ and $y=0$ planes). In addition, the red boxes in the insets report a plastic model obtained by a $3 \mathrm{D}$ printer for educational purposes. In particular, we report the complete 3D RI distribution, and Supplementary Movie 1 display slice-by-slice the tomogram reconstructions and the inner RI map corresponding to the hemoglobin distribution. Figure 2a shows an abnormal RBC detected in the healthy sample, and a one-side concavity is present resembling the shape typically observed in hereditary stomatocytosis. Figure $2 \mathrm{~b}$ shows one of the RBCs analyzed for the healthy sample in hypertonic solution, where the shape modification, as induced by the medium, provides the typical burr shape. We compare real cases with the simulated one (Supplementary Information) by means of the healthiness parameter, $H$, that is, the correlation coefficient between the measured and ideal 3D RI distributions. We assume that for $0.9 \leqslant H \leqslant 1$, a RBC can be considered normal; otherwise, the deviation from the discocyte shape is not negligible. $H$ values are reported for all of the cases presented in Figure 2 together with other global morphometric parameters, namely, the average RI, bio-volume $(V)$ and $\mathrm{CH}$ (see Supplementary Information for details).

To verify the accuracy of the proposed approach, we have tested the R-QPM for two highly diffused blood disorders. The first sample, as shown in Figure 2c, is from a patient affected with iron refractory iron deficiency anemia (IRIDA) caused by mutations in the TMPRSS6 gene (L63Pfs13-W590R in compound heterozygosity) ${ }^{42}$. The second sample, in Figure $2 \mathrm{~d}$, is from a patient affected with alpha-thalassemia caused by a heterozygous deletional event of both in-cis HBA1 genes (-CAMPANIA in heterozygosity) ${ }^{43}$. CBC reveals that MCVs are equal to 62.6 and $67.5 \mathrm{fl}$ and that $\mathrm{MCHs}$ are 18.5 and $21 \mathrm{pg}$ for the two patients, respectively. (Analysis performed by DAI.Med.Lab AOU Federico II University, Naples, Italy). In the case of anemia caused by an inherited defect in iron metabolism and thalassemia, the $\mathrm{CH}$ and $V$ values are in good agreement with the analysis reported in the literature and, by comparison, with the $\mathrm{CBC}$ from patients with a similar genotype ${ }^{42-44}$. Under our experimental conditions, the deformations of RBCs are negligible compared to the rotations they undergo, and the approximation of a rigid body is achieved ${ }^{36,45}$ (see Materials and methods for details).

The second class of cells we investigated are DAs. These cells have a much more complex inner structure 26,27 . As a test case, we analyzed the Skeletonema marinoi and the Thalassiosira rotula diatoms, as shown in Figure 3 and Supplementary Movies 5 and 6. Figure 3a and 3e shows two mirror QPMs recovered by SCC maximization. The retrieved 3D RI distributions of diatoms are depicted in Figure $3 \mathrm{~b}$ and $3 \mathrm{f}$, where the external shape, labeled in green, is used to identify the whole occupied volume. The fascinating result for DAs is that by choosing the proper RI threshold, the tomographic algorithm reveals the real shape and dimensions of the chloroplasts and their location (Figure $3 \mathrm{c}$ and $3 \mathrm{~g}$ ), in perfect agreement with the corresponding fluorescence images (Figure $3 \mathrm{~d}$ and $3 \mathrm{~h}$ ). In other words, the fluorescence acquisition or labeling of cells is, in principle, no longer necessary for R-TPM.

The corresponding calculated volumes are $V_{\text {tot }}=183.2 \mu \mathrm{m}^{3}$ and $V_{\text {chlor }}=38.7 \mu \mathrm{m}^{3}$ for the Skeletonema, $V_{\text {tot }}=1105.8 \mu \mathrm{m}^{3}$ and 

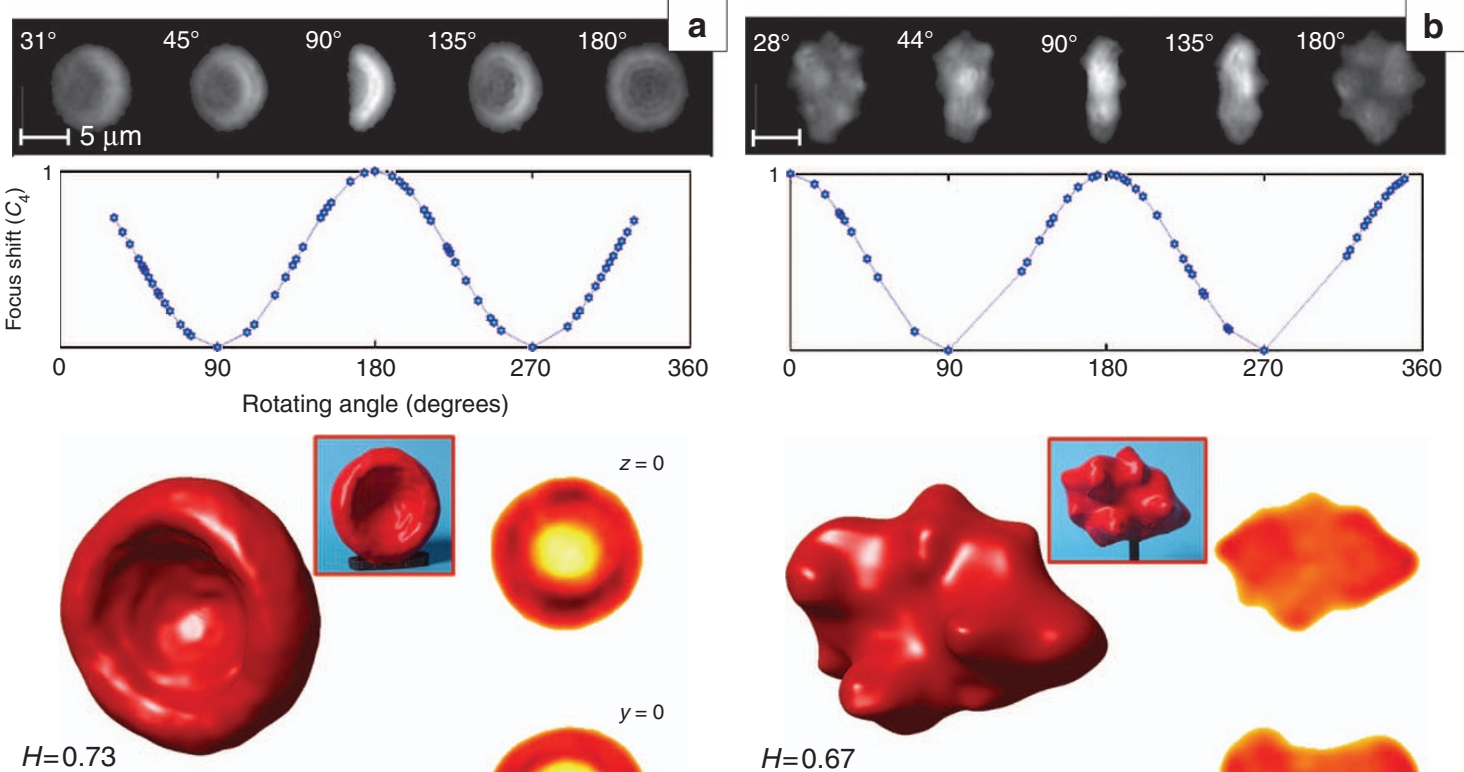

$H=0.73$

$\mathrm{ARI}=1.396$

$V=110.5 \mu \mathrm{m}^{3}$

$\mathrm{CH}=33.7 \mathrm{pg}$
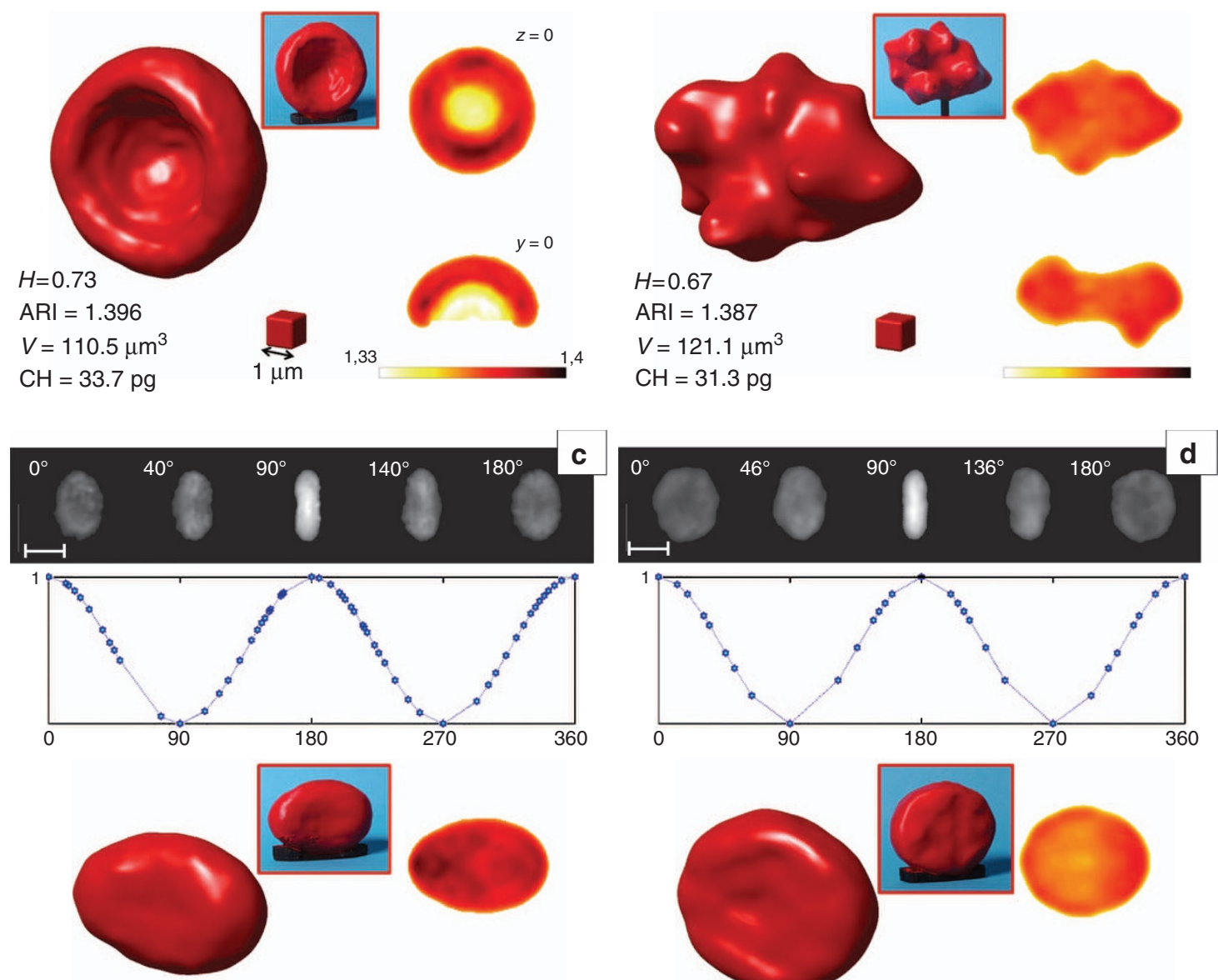

$H=0.72$

$\mathrm{ARI}=1.398$
$V=56.6 \mu \mathrm{m}^{3}$
$\mathrm{CH}=18.7 \mathrm{pg}$

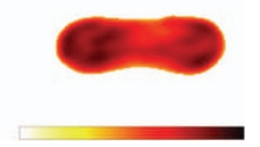

\section{$H=0.88$}

$\mathrm{ARI}=1.379$

$V=80.2 \mu \mathrm{m}^{3}$

$\mathrm{CH}=18.2 \mathrm{pg}$

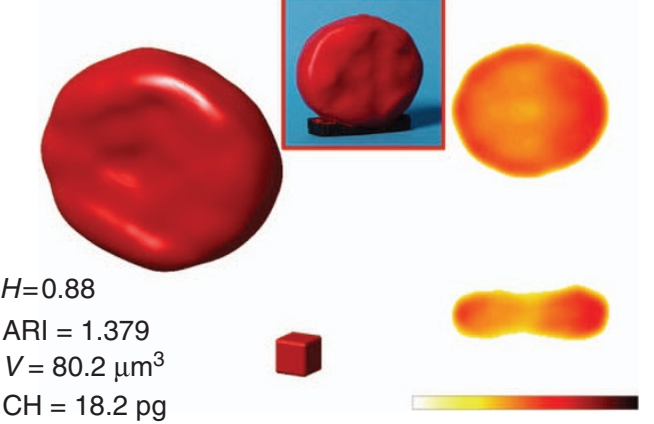

Figure 2 R-TPM for RBCs. (a-d) Results of R-TPM, applied on RBCs presenting morphological anomalies $(H<0.9)$ with respect to the ideal healthy one: (a) one-side concavity, (b) speculated, (c) iron deficiency anemic and (d) thalassemic RBC. For each RBC, we report the QPIs and the mathematical dependence of the defocus coefficient from the rotation angle and the tomogram retrieved by the QPIs and the RI distributions at the $z=0$ and $y=0$ planes. ARI, $V$ and $\mathrm{CH}$ are also reported together with the plastic 3D representations realized by a $3 \mathrm{D}$ printer. The maximum measured standard deviation of the RI obtained by this technique is 0.003 .

$V_{\text {chlor }}=100.1 \mu \mathrm{m}^{3}$ for Thalassiosira. Note that the chloroplasts (which correspond to the fluorescence part of the cell) represent only a fraction of the entire volume. The tomographic reconstruction allows us to exactly identify their location and dimensions. This achievement is a key point as variations in chloroplast shape and location may be used in diagnostics at the genus level. Moreover, diatom chloroplasts are the main targets of some water contaminants, such as fluoranthene, which is one of the principal constituent of $\mathrm{PAH}-\mathrm{contaminated}$ aquatic systems, and copper. It has been demonstrated that in the presence of these elements, chloroplasts show signs of structural rupture or even disintegration. Consequently, disturbances in the chloroplasts' integrity could lead to an inhibition of photosynthesis, thus leading to a reduction in the amount of energy that is available to the cells. Due to structural damage, the function of 


\section{Skeletonema marinoi}
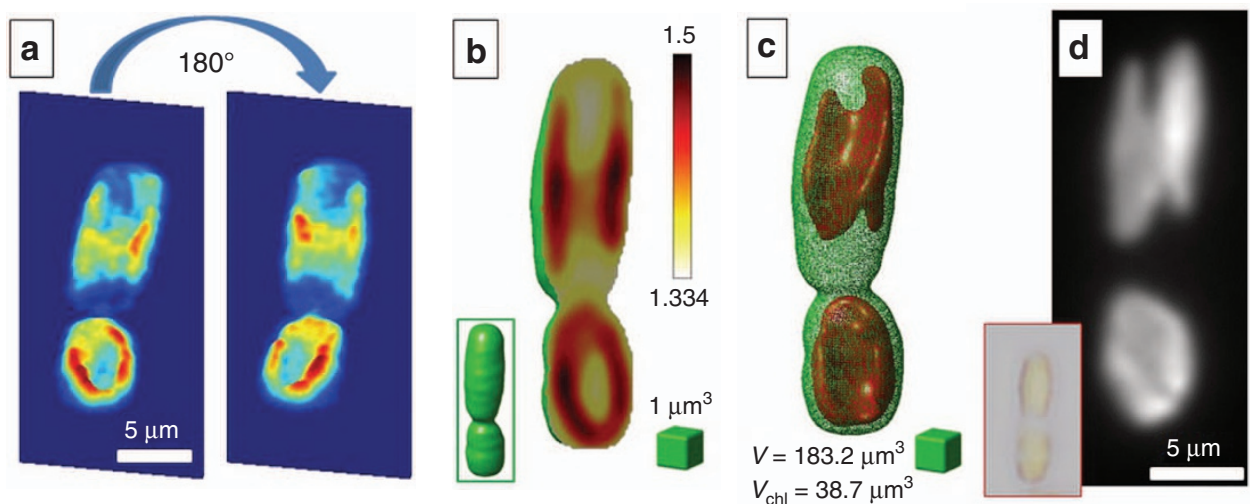

Thalassiosira rotula
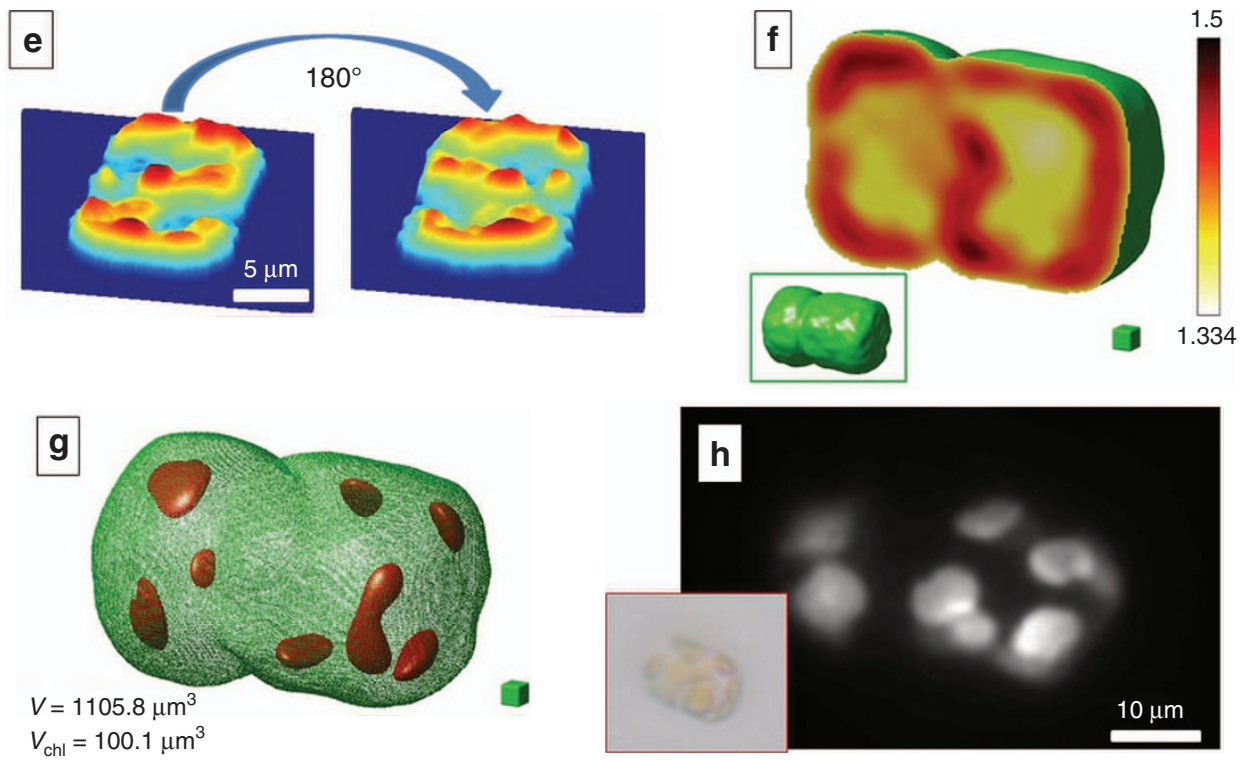

Figure 3 R-TPM for DA. (a-d) Skeletonema marinoi and (e-h) Thalassiosira rotula. (a, e) Two mirror QPMs used to define the rotating angle retrieval rule. (b, f) Calculated tomograms, where the central slices are shown (the complete tomographic scan is provided in Supplementary Movies 5 and 6). Inset figures in the green boxes show the whole tomogram shapes, highlighting the volume occupied by diatoms given by the parameter $V$. (c, $\mathbf{g})$ are obtained from (b, $\mathbf{f}$ ), respectively, by applying a RI threshold to extract the 3D distribution of the chloroplasts and their volume $\left(V_{\text {chl }}\right)$. (d, h) Fluorescence images used for comparison. Inset figures in the red boxes show the typical bright-field microscope images of the two diatoms.

the chloroplast may be affected; hence, algal cells will not be able to produce sufficient energy for growth and reproduction ${ }^{27}$. As a result, the abundance of diatoms is commonly considered a valid indicator of ecosystem health.

\section{CONCLUSIONS}

In conclusion, the R-TPM approach is a single-cell analysis tool that allows the study of hundreds of cells and supplies as output their complete morphologic classification in $3 \mathrm{D}$ via the self-rotation of cells in microfluidic channels. Technological improvement obtained in the practical implementation of tomography is challenging because the wavefront analysis of the sample along different directions intrinsically carries not only QPM information on the OPL of the cell but also information about the rotation angles. An intelligent numerical process can easily extract the data of angles, thus allowing accurate phase-contrast cyto-tomography. This avoids cumbersome calibration and the use of any mechanical and/or optoelectronic device to control the rotation, thereby enormously simplifying the experimental system compared with the current state of the art ${ }^{4,5,8,21}$. By exploiting this concept, we have shown that it is possible to furnish a full 3D RI distribution for many types of cells flowing along a microchannel with a relatively simple internal RI distribution. Similar algorithms are under study to adapt the technique to more general cases, such as inhomogeneous RI cells with little symmetry.

The technique has been proved for cells with different shapes and characteristics, such as RBCs and DA. The R-TPM is simple, quick and reliable. A route for full screening at the single-cell level can be a quite challenging achievement by means of the proposed approach. Applications are foreseen, for example, in the isolation and characterization of 'foreign' cells in the blood stream and in revealing specific oceanic contaminants that threaten the ecosystem via the analysis of damaged chloroplasts.

\section{CONFLICT OF INTEREST}

The authors declare no conflict of interest. 
1. Mertz J. Optical sectioning microscopy with planar or structured illumination. Nat Methods 2011; 8: 811-819.

2 Huang D, Swanson EA, Lin CP, Schuman JS, Stinson WG et al. Optical coherence tomography. Science 1991; 254: 1178-1181.

3 Yi J, Liu WZ, Chen SY, Backman V, Sheibani N et al. Visible light optical coherence tomography measures retinal oxygen metabolic response to systemic oxygenation. Light Sci Appl 2015; 4: e334, doi:10.1038/lsa.2015.107.

4 Choi W, Fang-Yen C, Badizadegan K, Oh S, Lue N et al. Tomographic phase microscopy. Nat Methods 2007; 4: 717-719

5 Kim K, Kim KS, Park H, Ye JC, Park YK. Real-time visualization of 3-D dynamic microscopic objects using optical diffraction tomography. Opt Express 2013; 21: 32269-32278

6 Charrière F, Marian A, Montfort F, Kuehn J, Colomb T et al. Cell refractive index tomography by digital holographic microscopy. Opt Lett 2006; 31: 178-180.

7 Kim T, Zhou RJ, Mir M, Babacan SD, Carney PS et al. White-light diffraction tomography of unlabelled live cells. Nat Photon 2014; 8: 256-263.

8 Mir M, Tangella K, Popescu G. Blood testing at the single cell level using quantitative phase and amplitude microscopy. Biomed Opt Express 2011; 2: 3259-3266.

9 Kim Y, Shim H, Kim K, Park H, Jang S et al. Profiling individual human red blood cells using common-path diffraction optical tomography. Sci Rep 2014; 4: 6659.

10 Sung $Y$, Lue N, Hamza B, Martel J, Irimia D et al. Three-dimensional holographic refractive-index measurement of continuously flowing cells in a microfluidic channel. Phys Rev Appl 2014; 1: 014002.

11 Yoon J, Kim K, Park H, Choi C, Jang S et al. Label-free characterization of white blood cells by measuring 3D refractive index maps. Biomed Opt Express 2015; 6 : 3865-3875.

12 Bishara W, Zhu HY, Ozcan A. Holographic opto-fluidic microscopy. Opt Express 2010; 18: 27499-27510.

13 Isikman SO, Bishara W, Zhu HY, Ozcan A. Optofluidic tomography on a chip. Appl Phys Lett 2011; 98: 161109.

14 Isikman SO, Bishara W, Mavandadi S, Yu FW, Feng S et al. Lens-free optical tomographic microscope with a large imaging volume on a chip. Proc Natl Acad Sci USA 2011; 108: 7296-7301.

15 Kamilov US, Papadopoulos IN, Shoreh MH, Goy A, Vonesch C et al. Learning approach to optical tomography. Optica 2015; 2: 517-522.

16 Psaltis D, Quake SR, Yang C. Developing optofluidic technology through the fusion of microfluidics and optics. Nature 2006; 442: 381-386.

17 Pégard NC, Toth ML, Driscoll M, Fleischer JW. Flow-scanning optical tomography. Lab Chip 2014; 14: 4447-4450.

18 Padgett M, Di Leonardo R. Holographic optical tweezers and their relevance to lab on chip devices. Lab Chip 2011; 11: 1196-1205

19 Merola F, Miccio L, Memmolo P, Di Caprio G, Galli A et al. Digital holography as a method for 3D imaging and estimating the biovolume of motile cells. Lab Chip 2013, 13: $4512-4516$.

20 Memmolo P, Miccio L, Merola F, Gennari O, Netti PA et al. 3D morphometry of red blood cells by digital holography. Cytometry A 2014; 85: 1030-1036.

21 Habaza M, Gilboa B, Roichman Y, Shaked NT. Tomographic phase microscopy with $180^{\circ}$ rotation of live cells in suspension by holographic optical tweezers. Opt Lett 2015 40: 1881-1884.

22 Heng X, Erickson D, Baugh LR, Yaqoob Z, Sternberg PW et al. Optofluidic microscopya method for implementing a high resolution optical microscope on a chip. Lab Chip 2006; 6: 1274-1276.

23 Miccio L, Memmolo P, Merola F, Netti PA, Ferraro P. Red blood cell as an adaptive optofluidic microlens. Nat Commun 2015; 6: 6502

24 Elgsaeter A, Stokke BT, Mikkelsen A, Branton D. The molecular basis of erythrocyte shape. Science 1986; 234: 1217-1223.

25 Ford J. Red blood cell morphology. Int J Lab Hematol 2013; 35: 351-357.
26 Bedoshvili YD, Popkova TP, Likhoshway YV. Chloroplast structure of diatoms of different classes. Cell Tiss Biol 2009; 3: 297-310.

27 Wang LP, Zheng BH. Toxic effects of fluoranthene and copper on marine diatom Phaeodactylum tricornutum. J Environ Sci 2008; 20: 1363-1372.

28 Arpali SA, Arpali C, Coskun AF, Chiang HH, Ozcan A. High-throughput screening of large volumes of whole blood using structured illumination and fluorescent on-chip imaging. Lab Chip 2012; 12: 4968-4971.

29 Uspal WE, Burak Eral H, Doyle PS. Engineering particle trajectories in microfluidic flows using particle shape. Nat Commun 2013; 4: 2666.

30 D'Avino G, Maffettone PL. Particle dynamics in viscoelastic liquids. J Non-Newtonian Fluid Mech 2015; 215: 80-104.

31 Amini H, Lee W, Di Carlo D. Inertial microfluidic physics. Lab Chip 2014; 14 2739-2761.

32 Pham HV, Bhaduri B, Tangella K, Best-Popescu C, Popescu G. Real time blood testing using quantitative phase imaging. PLoS One 2013; 8: e55676.

33 Merola F, Memmolo P, Miccio L, Bianco V, Paturzo M et al. Diagnostic tools for lab-onchip applications based on coherent imaging microscopy. Proc IEEE 2015; 103: 192-204.

34 Cotte $\mathrm{Y}$, Toy MF, Depeursinge $\mathrm{C}$. Beyond the lateral resolution limit by phase imaging. $J$ Biomed Opt 2011; 16: 106007.

$35 \mathrm{Kim}$ MK, Yu LF, Mann CJ. Interference techniques in digital holography. J Opt A Pure Appl Opt 2006; 8: S518.

36 Villone MM, D'Avino G, Hulsen MA, Maffettone PL. Dynamics of prolate spheroidal elastic particles in confined shear flow. Phys Rev E 2015; 92: 062303.

37 Sharpe J, Ahlgren U, Perry P, Hill B, Ross A et al. Optical projection tomography as a tool for 3D microscopy and gene expression studies. Science 2002; 296: 541-545.

38 Dardikman G, Habaza M, Waller L, Shaked NT. Video-rate processing in tomographic phase microscopy of biological cells using CUDA. Opt Express 2016; 24 11839-11854.

39 Memmolo P, Miccio L, Paturzo M, Di Caprio G, Coppola G et al. Recent advances in holographic 3D particle tracking. Adv Opt Photon 2015; 7: 713-755.

40 Memmolo P, lannone M, Ventre M, Netti PA, Finizio A et al. On the holographic 3D tracking of in vitro cells characterized by a highly-morphological change. Opt Express 2012; 20: 28485-28493.

41 Memmolo P, Distante C, Paturzo M, Finizio A, Ferraro P et al. Automatic focusing in digital holography and its application to stretched holograms. Opt Lett 2011; 36: 1945-1947.

42 De Falco L, Silvestri L, Kannengiesser C, Morán E, Oudin C et al. Functional and clinical impact of novel TMPRSS6 variants in iron-refractory iron-deficiency anemia patients and genotype-phenotype studies. Hum Mutat 2014; 35: 1321-1329.

43 Sessa R, Puzone S, Ammirabile M, Piscopo C, Pagano L et al. Identification and molecular characterization of the-CAMPANIA deletion, a novel $a^{\circ}$-thalassemic defect, in two unrelated Italian families. Am J Hematol 2010; 85: 143-144.

44 Origa R, Moi P, Galanello R, Cao A. Alpha-thalassemia. In: Pagon RA et al. editors. GeneReviews. Seattle, WA: University of Washington; 2013.

45 Fedosov DA, Peltomäki M, Gompper G. Deformation and dynamics of red blood cells in flow through cylindrical microchannels. Soft Matter 2014; 10: 4258-4267.

(c) $(\$$ This work is licensed under a Creative Commons Attributionc. $\mathrm{BY}$ NC ND NonCommercial-NoDerivs 4.0 International License. The images or other third party material in this article are included in the article's Creative Commons license, unless indicated otherwise in the credit line; if the material is not included under the Creative Commons license, users will need to obtain permission from the license holder to reproduce the material. To view a copy of this license, visit http:// creativecommons.org/licenses/by-nc-nd/4.0/

C) The Author(s) 2017

Supplementary Information for this article can be found on the Light: Science \& Applications' website (http://www.nature.com/lsa). 